\title{
Estimation of height changes of GNSS stations from the solutions of short vectors and PSI measurements
}

\author{
Jan Krynski*, Lukasz Zak, Dariusz Ziolkowski, Jan Cisak, \\ Magdalena Lagiewska \\ Institute of Geodesy and Cartography \\ Centre of Geodesy and Geodynamics \\ 27 Modzelewskiego St., 02-679 Warsaw, Poland \\ e-mails: jan.krynski@igik.edu.pl; lukasz.zak@igik.edu.pl,dariusz.ziolkowski@igik.edu.pl, \\ jan.cisak@igik.edu.pl, magdalena.lagiewska@igik.edu.pl \\ * Corresponding author: Jan Krynski
}

\begin{abstract}
Time series of weekly and daily solutions for coordinates of permanent GNSS stations may indicate local deformations in Earth's crust or local seasonal changes in the atmosphere and hydrosphere. The errors of the determined changes are relatively large, frequently at the level of the signal.

Satellite radar interferometry and especially Persistent Scatterer Interferometry (PSI) is a method of a very high accuracy. Its weakness is a relative nature of measurements as well as accumulation of errors which may occur in the case of PSI processing of large areas. It is thus beneficial to confront the results of PSI measurements with those from other techniques, such as GNSS and precise levelling.

PSI and GNSS results were jointly processed recreating the history of surface deformation of the area of Warsaw metropolitan with the use of radar images from Envisat and CosmoSkyMed satellites. GNSS data from Borowa Gora and Jozefoslaw observatories as well as from WAT1 and CBKA permanent GNSS stations were used to validate the obtained results. Observations from 2000-2015 were processed with the Bernese v.5.0 software. Relative height changes between the GNSS stations were determined from GNSS data and relative height changes between the persistent scatterers located on the objects with GNSS stations were determined from the interferometric results.

The consistency of results of the two methods was 3 to 4 times better than the theoretical accuracy of each. The joint use of both methods allows to extract a very small height change below the level of measurement error.
\end{abstract}

Keywords: height changes, GNSS, Persistent Scatterer Interferometry 


\section{Introduction}

GNSS and Persistent Scatterer Interferometry (PSI) are two totally independent geometric observation techniques which can be applied for the determination of height variations of the objects on the Earth's surface. They substantially differ in source data used, acquiring and processing methods, spatial distribution and temporal resolution of the results. They also differ in terms of certainty of getting the correct results with the specified accuracy, in particular in the case of data analysis in large distances from the interferometric point of view. Also this latter term has a different meaning in the case of both methods. The results of PSI measurements have usually rather local character. The spatial extent of interferometric results usually does not exceed the range of tens of kilometres from the reference point of phase unwrapping. This distance considered to be very large for interferometric measurements is usually the distance between the nearest permanent GNSS stations and is defined as a short vector in this second method.

Deformation data obtained from processing GNSS observations at permanent GNSS stations exhibit very small spatial resolution (determined by the distribution of permanent GNSS stations) as compared with the spatial resolution of PSI results but high temporal resolution (most frequently 24 hours). High accuracy (at the level of $1 \mathrm{~cm}$ ) of the determination of relative height variations independently on the distance between stations is a huge advantage of the GNSS method. Appropriate GNSS data processing provides a reliable result with no necessity of its validation with the use of other observation techniques. Deformation data obtained from PSI exhibit very high spatial resolution (even up to a several thousand observations in square kilometre) but smaller temporal resolution (recently ca 8 days). If the PSI measurements performed on large areas, additionally characterized by dynamic weather conditions, resulted in very high spatial variability of radar signal delay in the atmosphere, the phase unwrapping errors can occur during the processing of the data. This is particularly evident on the areas were non-linear deformations of large spatial extent occur, which may result that statistical behaviour in space and time of interferometric phase related to the deformation is very similar to the one triggered by Atmospheric Phase Screen. In such situations it is highly recommended to validate PSI results with the use of other methods, e.g. GNSS, although theoretical accuracy of the determination of height variations with PSI which is higher than with GNSS, and in the case of favourable conditions when it might even be at the level of 1-2 mm, cannot always be reached. Different character of those two observation techniques, in particular different advantages and disadvantages in their use to the same purpose, make them complementary. Their common use might be beneficial for each of them, in particular in terms of increasing reliability of the results. The objective of this work is to show that PSI data can be used to increase reliability and accuracy of height variations of permanent GNSS stations determined from GNSS data in case of correct processing of the phase unwrapping in large areas. Integration of both techniques may provide height variation signal below the noise of GNSS solutions. 


\section{Methods}

\subsection{Precise determination of height changes from GNSS data}

Processing observations from permanent GNSS stations provides most accurate height variations which can be determined with the use of the GNSS technique. Permanent GNSS stations with long data records play an important role in monitoring deformations (Cisak et al., 2011).

IGS and EPN permanent GNSS stations networks are used for the realization of the International Terrestrial Reference Frame (ITRF) and the European Terrestrial Reference Frame (ETRF). Data acquired at permanent GNSS stations are processed in Local Analysis Centres (LACs) providing weekly and daily solutions for station coordinates. Those solutions may indicate local deformations in the Earth' crust (active seismicity areas) or local seasonal changes in the atmosphere and hydrosphere; the errors of their determination are frequently at the level of the signal determined. Variations of station coordinates averaged over yearly intervals are used for the determination of station velocities in a global reference frame, and even in continental reference frames, e.g. ETRF.

Analyses of the results of GNSS observations at permanent stations of IGS and EPN networks as well as regional networks indicate very high accuracy of determined variations of station coordinates. Height variations are obtained from network solutions with centimetre accuracy (Bruyninx et al., 2011, http://www. epncb.oma.be/) but from the solutions of short vectors referred to the reference station - with accuracy at the level of single millimetres (Krynski and Zanimonskiy, 2005; Godah et al., 2012).

Example of height variations from daily and weekly solutions for JOZE station from the short BOGO - JOZE vector with BOGO as reference station, is shown in Figure 1. Weekly solutions for height variations of JOZE station from the EPN network have been compared with the respective ones from the short vector BOGO - JOZE with the reference station BOGO (Figure 2). Their statistics are given in Table 1.

Deformations determined with the use of the GNSS technique refer only to GNSS stations providing observations. Since the number of such stations in the area investigated is limited, deformation data exhibit a discreet set of low resolution. 


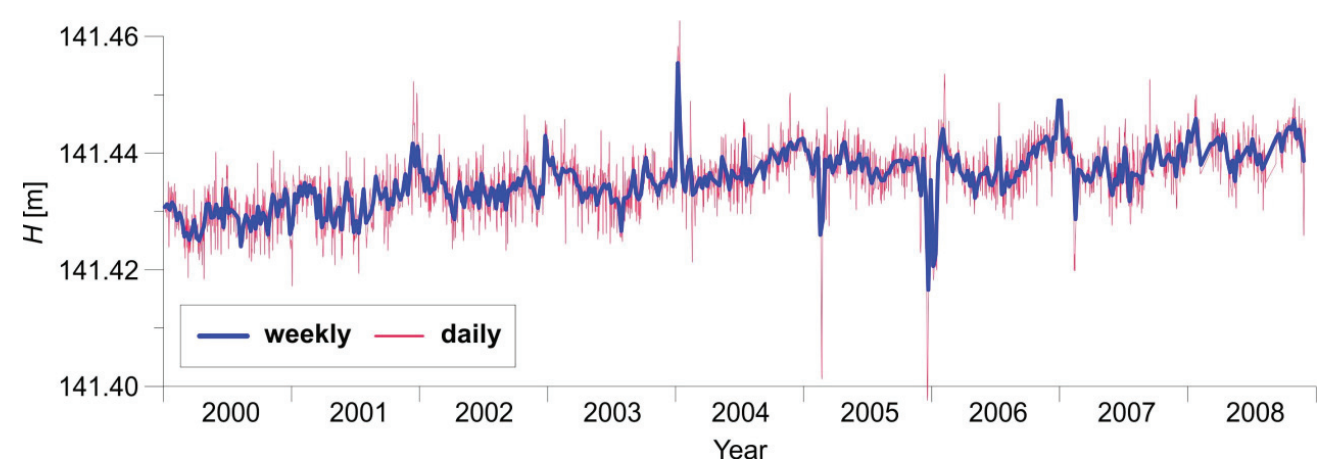

Fig. 1. Example of height variations of JOZE station from daily and weekly solutions of the short vector BOGO - JOZE with the reference station BOGO

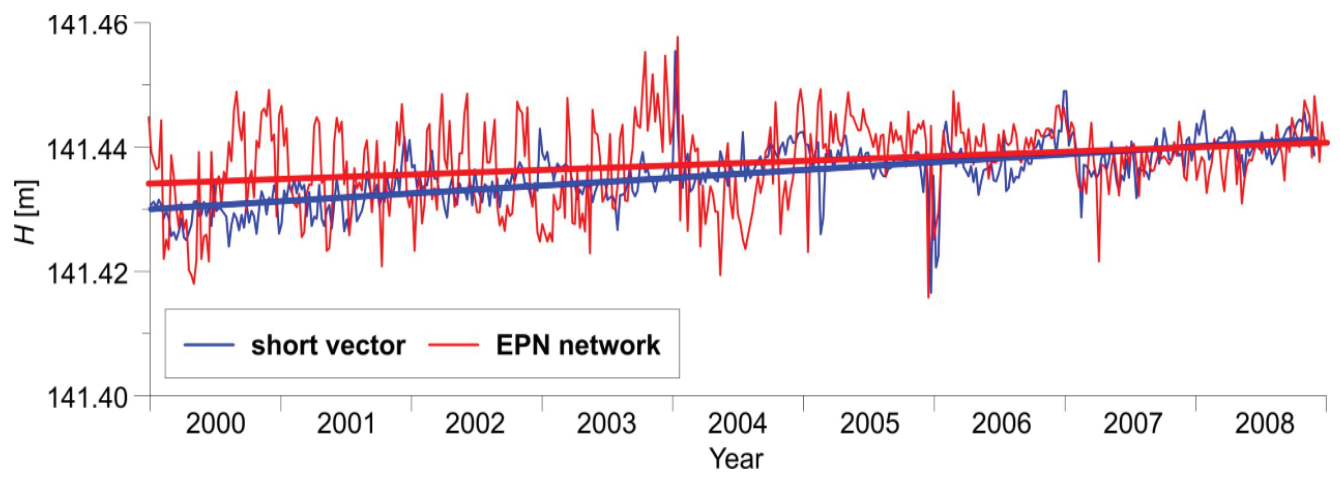

Fig. 2. Weekly solutions for height variations of JOZE station from the EPN network and the short vector BOGO - JOZE with the reference station BOGO

Table 1. Statistics of EPN solutions and short vector solutions for JOZE station [m]

\begin{tabular}{|l|c|c|c|}
\hline & Mean & Max - Min & Std. dev. \\
\hline EPN solution & 141.437 & 0.042 & 0.007 \\
\hline Short vector weekly solution & 141.436 & 0.039 & 0.005 \\
\hline Short vector daily solution & 141.436 & 0.065 & 0.006 \\
\hline
\end{tabular}




\subsection{Persistent Scatterer Interferometry}

Persistent Scatterer Radar Interferometry (PSI) belongs to the group of multitemporal interferometric methods. It is mainly applied for examining slow ground deformations using satellite data acquired from imaging SAR sensors. The radar interferometry (InSAR) was first time used for Earth surface studies in 1988, in order to generate Digital Terrain Model (DTM) (Zebker and Goldstein, 1986). Next, at the beginning of nineties, its modification - differential interferometry (DInSAR) started to be applied for examining slow ground deformations (Massonet et al., 1993; Zebker et al., 1994). Due to the limitation of this method, caused by so-called temporal decorrelation, the best results were achieved mainly for dry, semi-dry and rocky areas, characterized by high temporal stability of land cover. The turning point was the development of the Persistent Scatterer Interferometry (PSI) (Feretti et al., 2000, 2001). In this method solely objects characterized by high stability of reflection and high phase coherence in a long period (persistent scatterers) are used. In a temperate zone mainly anthropogenic objects fulfil these conditions.

Studies of ground deformations with the use of interferometric methods are possible due to expressing changes of the distance between the ground and the satellite in two different time periods as a part of the wavelength recorded by a satellite sensor. Since the sensors record only the phase of the last phase cycle, the method enables to determine solely relative changes of height within the study area, and accuracy of final results is strictly related to the correctness of so-called phase unwrapping. In this process, for each pixel representing persistent scatterer the measured phase is related to the defined phase cycle. Determination of relative number of phase cycles 2Pi for each pixel is not easy, as interferometric phase is affected, apart from deformations, by other factors, such as terrain relief, signal delay in the atmosphere, change of land cover and change of dielectric constant. The latter factor generates random noise, called temporal decorrelation. In order to determine correctly ground deformations by interferometric methods, one should well separate signal phase component responsible for terrain deformation from the remaining components. The process is done using methods of statistical modelling. In order to obtain the results of ground deformation by the PSI method minimum 20-25 radar images recorded from the same orbit but at different time are needed. As a result of processing radar images with the use of PSI method two products are generated: mean deformation at a given time period defined in $\mathrm{mm} /$ year (Figure 3 ) and deformation history for each point identified as a persistent scatterer, which demonstrates height changes in relation to the zero epoch (Figure 4).

Accuracy of the results obtained with the use of this method can reach the level even below $1 \mathrm{~mm} /$ year for first product. In the case of deformation history it is assumed that the accuracy is usually lower and depends of the quality of persistent scaterrers and the character of the deformation: its linearity, rate etc. The mentioned accuracies are achieved under rather favourable conditions. Final accuracy of the results is affected by several factors, first of all by the resolution of images and radar wavelength, type of deformation (spatial extend, velocity and non-linearity in time), 
type of terrain and related quality of persistent scatterers, as well as correctness of the data processing. In the case of PSI measurements performed on large areas, one of the important factors which can lower final accuracy is a high spatial variability of the signal delay in the atmosphere which occurs on a large number of images in an interferometic stack which is a typical situation in the case of Central Poland located in a temperate climatic zone. Thus, it is highly recommended to validate the results obtained on large areas with the use of PSI method by independent GNSS measurements.

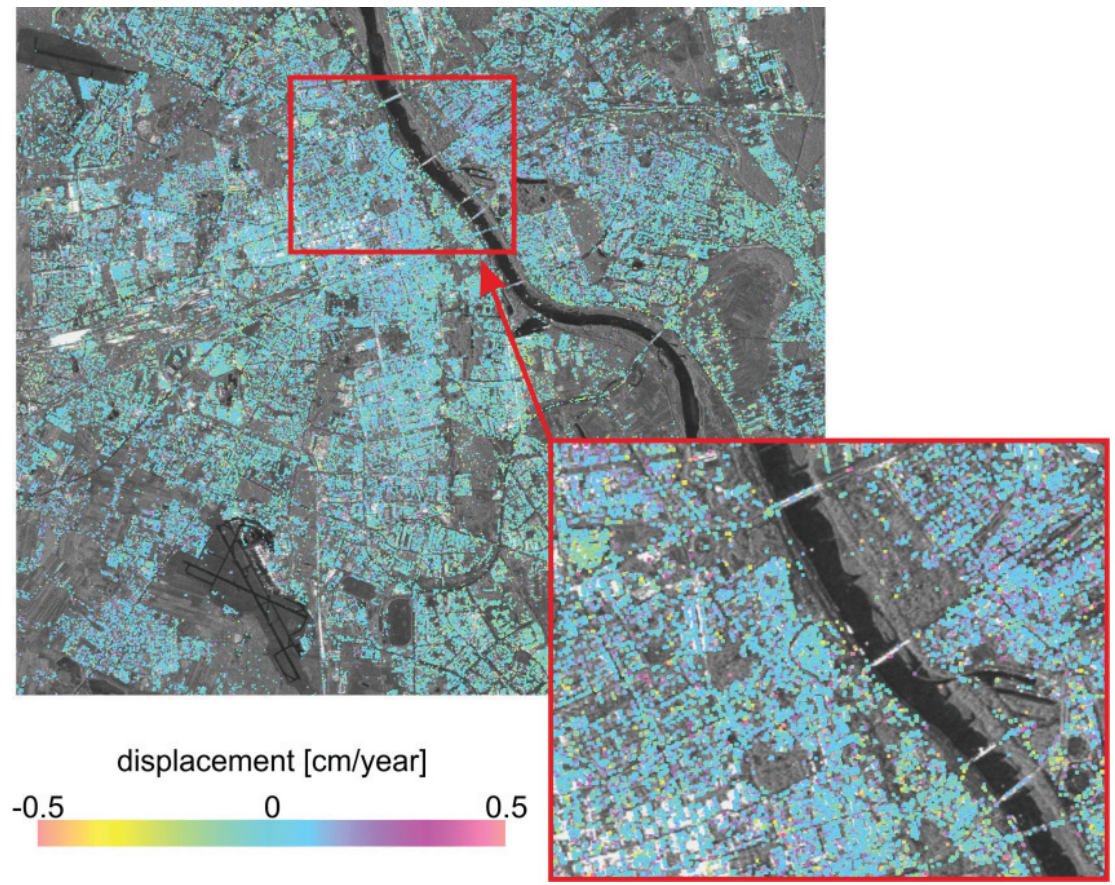

Fig. 3. The example of deformation map obtained as a result of processing radar images with the use of the PSI method

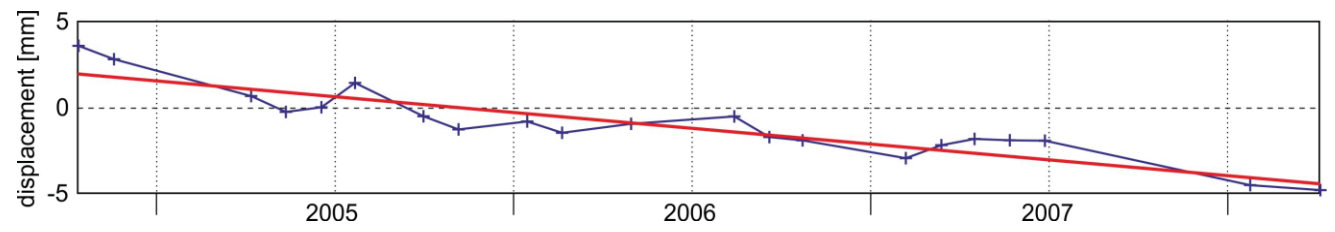

Fig. 4. The example of deformation history obtained as a result of processing radar images with the use of the PSI method 


\section{Study area and data}

\subsection{Study area}

The study area covers the Warsaw agglomeration. It is located in the central part of Mazowieckie Voivodship within the Warsaw Basin and cut across by the Vistula River valley (Figure 5). Numerous deformations associated probably mainly with the changes of hydrology within the Vistula River valley, Warsaw Escarpment and its surroundings are observed in the study area which is in principle geologically stable (Sawicki, 1960). Local deformations are associated with the construction of the metro line as well as with the impact of various other investments in the subsoil.

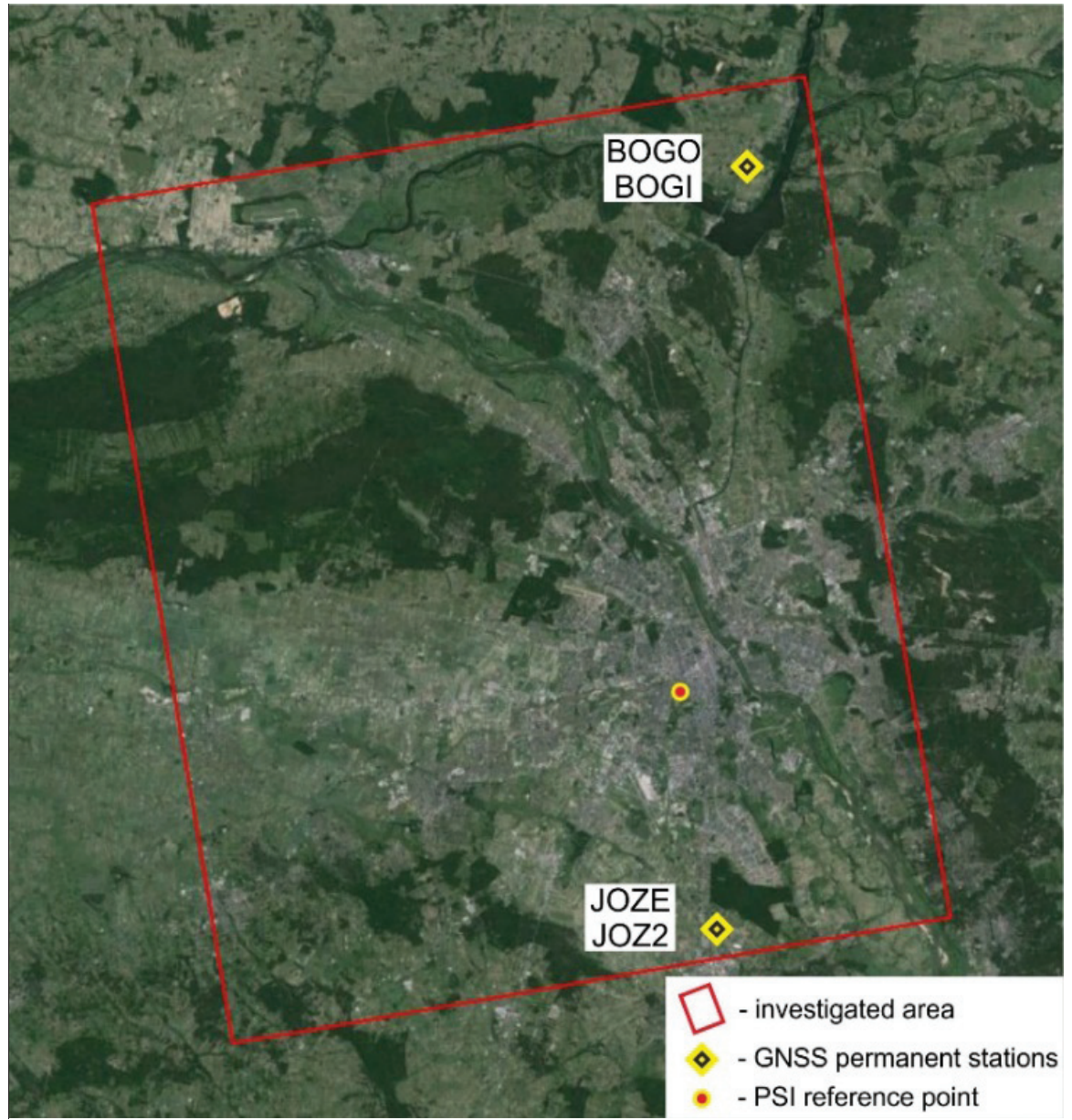

Fig. 5. Study area with location of GNSS stations 


\subsection{GNSS data}

GNSS data from 2000-2008 with sampling rate of 30 seconds from BOGO and BOGI stations at the Geodetic-Geophysical Observatory Borowa Gora of the Institute of Geodesy and Cartography, and JOZE, JOZ2 stations at the Astrogeodetic Observatory in Jozefoslaw of the Warsaw University of Technology (Bosy and Krynski, 2015) operating in the EUREF Permanent Network were used in this study.

Three vectors connecting BOGO station with BOGI, JOZE, and JOZ2 stations have been processed with the Bernese v.5.0 software (cf. Dach et al., 2007) using precise ephemeris and Earth rotation parameters provided by the International GNSS Service (IGS). Earth tides and ocean tide loading model coefficients based on IERS and FES2004 models (Lyard et al., 2006), respectively, were implemented to eliminate the tide-loading effects. Antenna Phase Centre Variations (PCV) were modelled with the use of the absolute PCV model.

Following the EPN Local Analysis Centres recommendations (see http://www. epncb.oma.be), an optimum strategy was applied to process the data in daily session solutions. The basic steps of this strategy can be described in brief as follows:

- The baselines were created in daily batches from single differencing files.

- Cycle slips were detected and corrected; outliers were detected and removed from the observations.

- Zenith path delays were estimated at $2 \mathrm{~h}$ intervals for baseline stations; their a priori values were obtained using the DRY NEILL model, and wet part of the troposphere was estimated with the use of the WET NEILL mapping function (Neill, 1996).

- The phase ambiguities have been resolved using the SIGMA-dependent strategy; both frequencies L1 and L2 were used, the minimum elevation angle was set to $3^{\circ}$, and the elevation-dependent weighting was applied by using the $\cos 2 z$ function.

- Each baseline was adjusted considering BOGO as a fix station $(X, Y, Z$ coordinates in ETRF2000 frame on epoch of observation).

Calculated $X, Y, Z$ coordinates of BOGI, JOZE, and JOZ2 stations have been transformed to ellipsoidal coordinates $(\varphi, \lambda, h)$ and then to planar coordinates in PL-2000 frame as well as normal height. Statistics of height variations of the stations investigated obtained from daily GNSS solutions for short vectors are given in Table 2.

Table 2. Statistics of heights variations of the stations investigated obtained from daily GNSS solutions for short vectors [m]

\begin{tabular}{|l|l|l|l|}
\hline Station & Mean & Max - Min & Std. dev. \\
\hline BOGI & 139.915 & 0.021 & 0.002 \\
\hline JOZE & 141.436 & 0.065 & 0.006 \\
\hline JOZ2 & 152.519 & 0.048 & 0.005 \\
\hline
\end{tabular}




\subsection{Radar data and PSI product}

29 radar images from ascending orbit A143 of Envisat satellite from 2004-2008 were used in the following research. Those images were recorded in $\mathrm{C}$ band of $5.5 \mathrm{~cm}$ wavelength and spatial resolution of $5 \mathrm{~m} \times 16 \mathrm{~m}$. Although nominal repeatability of Envisat satellite imaging is 35 days, intervals between registration of the individual images collected are in some cases larger due to the lack of acquisitions. In addition, several images have been removed from the set during processing due to the very large perpendicular baseline or very unfavourable weather conditions during the image registration. It was decided to remove images with a perpendicular baseline exceeding $600 \mathrm{~m}$ as they caused very significant reduction of Persistent Scatters as well as the images with the highest spatial variability of the signal delay in the atmosphere as they caused many phase unwrapping errors. Finally 21 radar images from the period 2004-10-14 - 2008-03-27 with intervals between them from 36 days to even 210 days (between 2007-06-21 and 2008-01-17) were analysed. The average frequency of data collection during the period analysed amounted to less than 60 days. The entire processing of radar images, including analysis using persistent scatterers interferometry was conducted with the use of Gamma Remote Sensing software. The map of average deformation per year for the whole area investigated and the history of deformations for each point being a persistent scatterer were developed as a result of the research. The reference epoch (master image) for the entire data set was the day of 2005-11-03. The reference point (starting point for phase unwrapping process) were selected in the city center in the Warsaw Filters because their area is considered very stable due to its geological structure (thick layers of clay). Its location in the centre of the investigated area minimizes also the problem of error propagation. Throughout the area of investigated Warsaw agglomeration more than one million of persistent scatterers were identified. Among others, they are on all objects on which all GNSS stations used in this study are located (Figure 6). Therefore, the results are not subject to errors which would occur if the solutions of PSI and GNSS methods are obtained from slightly different locations. Shown in Figure 6a shift of PSI points with respect to the GNSS station by a few meters towards the east is the result of spatial resolution of radar images and associated with it inaccuracies of the geometrization process. However, detailed analysis of objects which can be used as persistent scatterers in the PSI method in this area indicates that with no doubt these reflections come from the same building and the pillar on which the stations BOGO and BOGI are located. In the case of JOZ2 station a number of persistent scatterers located on the roof of the Observatory building and a few that are the result of a double reflection: the wall of the building - street were identified (Figure 6b). Identification of persistent scatterers for JOZE station located on the monumented pillar failed. 

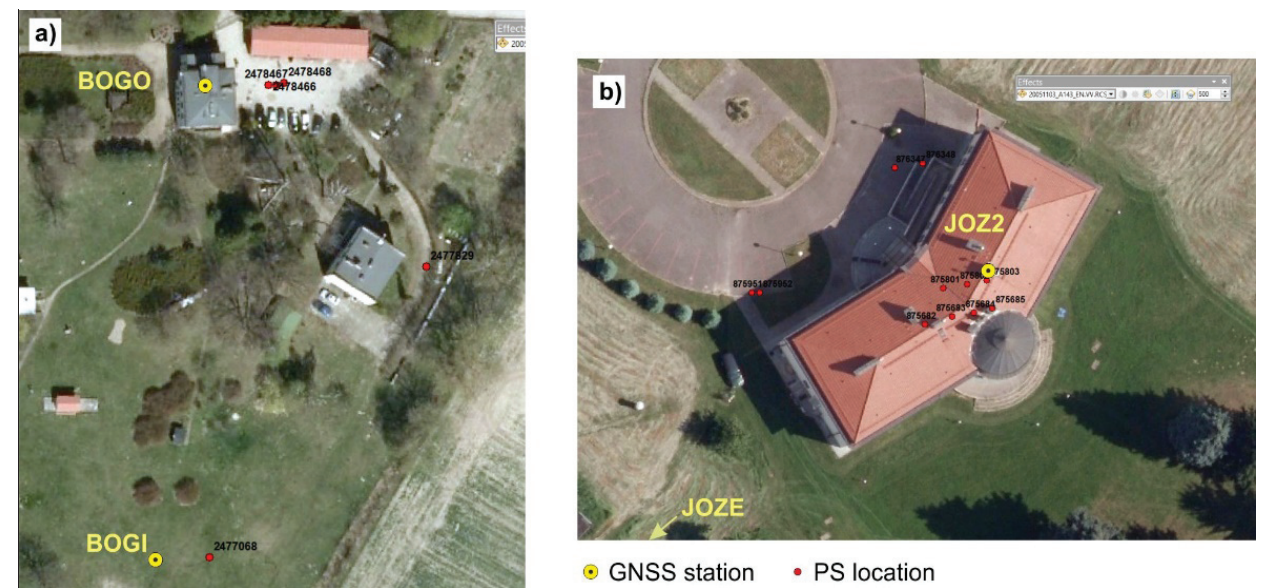

• GNSS station - PS location

Fig. 6. Persistent scatterers in the vicinity of BOGO and BOGI a) and JOZ2

b) permanent GNSS stations

\section{Methods of data integration}

The persistent scatterers located on the objects in the neighbourhood of GNSS stations BOGO, BOGI, JOZE, JOZ2 (Figure 6) were chosen from the processed PSI data. Deformations with respect to phase unwrapping point were obtained for each of those points for 21 days in the period of 2004-2008 (Figure 7).
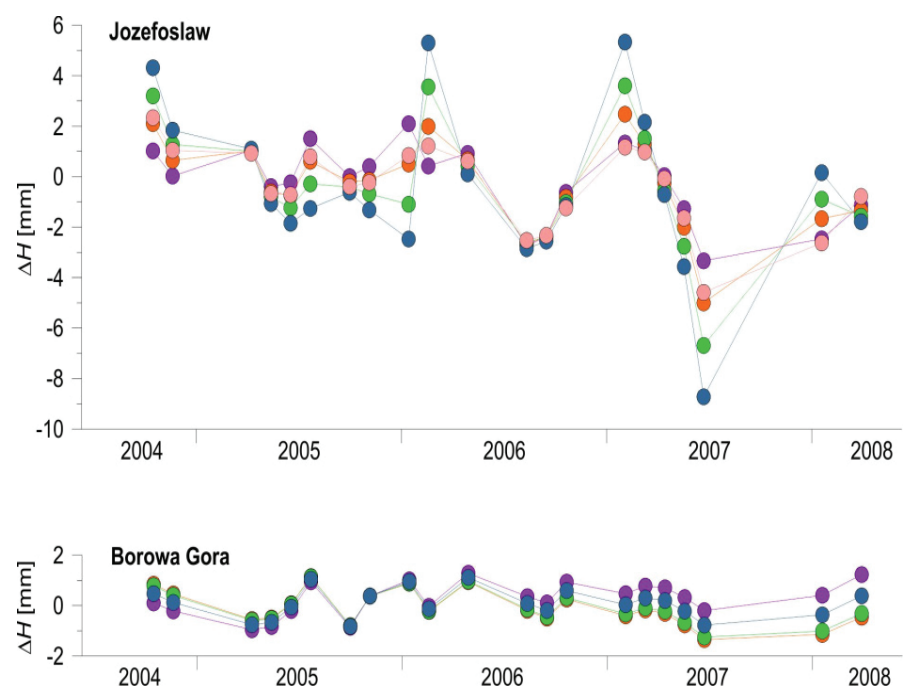

Fig. 7. Relative deformations in height component of Borowa Gora and Jozefoslaw form PSI data $[\mathrm{mm}]$ 
Two dependencies can clearly be observed. The discrepancies for individual persistent scatterers on the objects JOZ2 and BOGO are different in different dates; they range from less than $1 \mathrm{~mm}$ to about $5 \mathrm{~mm}$. The dispersion of the points on the object JOZ2 is for chosen dates several times larger than in the case of a BOGO object, where it does not exceed $2 \mathrm{~mm}$. The observed differences in the magnitudes of deformations for different points on the same object in different dates may be due to measurement inaccuracies resulting from many factors affecting the phase of the radar signal, and in this situation they will reflect the accuracy of the PSI method, or may be the result of real differences in the deformation of individual parts of the buildings. Based on the available data it is not possible to tell which of these two elements is critical. With high probability it comes to both factors. The first is an inherent feature of interferometric measurements. The second could be due to non-randomness of the scatter values for the individual points in time. For example, point Jozefoslaw indicated pink in Figure 7, exhibits throughout the course of time significantly smaller variability than the point marked in blue.

From the solutions for the vectors between the station BOGO and the stations BOGI, JOZE and JOZ2 from GNSS data the time series of heights of the endpoints of vectors with a daily resolution was determined. Then the results obtained were filtered off eliminating outliers (6\% observations) by more than $1 \mathrm{~cm}$ from the mean value of two adjacent.

In order to make possible comparison of results obtained with both methods described in the article, height variations of GNSS permanent stations were calculated in relation to the same period of reference (2005-11-03) as in the case of PSI solution (Figure 8) according to the following formula:

$$
\Delta H_{i}=H_{i}-H_{e 0}
$$

where $H_{i}$ is the height of GNSS station in epoch $i$, and $H_{e 0}$ is the height of GNSS station in the reference epoch.

Comparison of the results at this stage indicates their good agreement although they still differ in terms of reference point used. The reference point for the PSI method is the persistent scatterer located in the Warsaw Filters area (Figure 5). The reference point for GNSS results is BOGO station. In order to eliminate the effect of different reference points, relative difference in the vertical displacements between BOGI and JOZ2 stations obtained from GNSS and selected persistent scatterer corresponding to the permanent stations were calculated (Figure 9). The results obtained were used for comparison of the vertical displacements obtained from PSI and GNSS. 


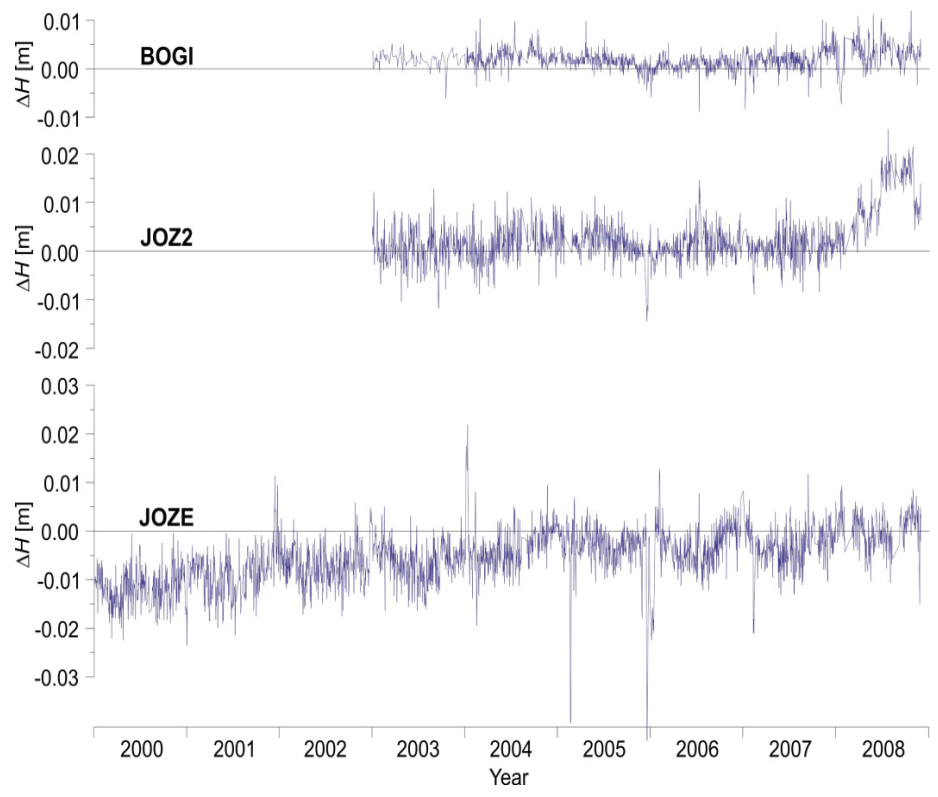

Fig. 8. Deformations in height component at BOGI, JOZ2 and JOZE relative to BOGO from GNSS data referred to epoch 2005-11-03

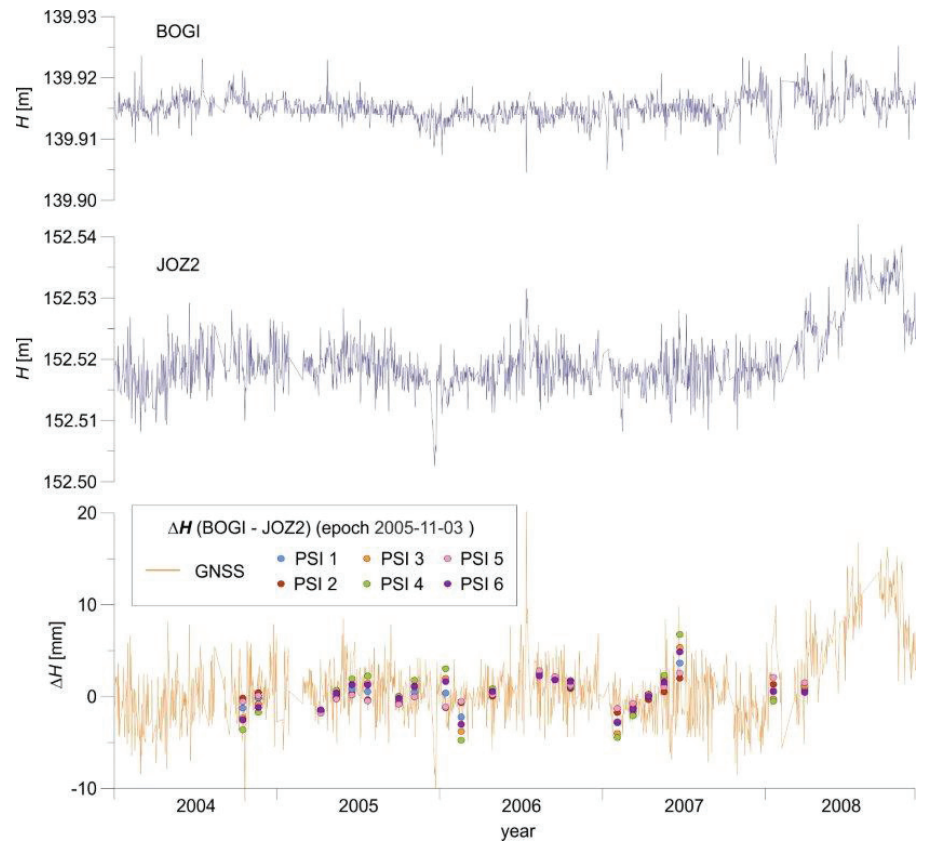

Fig. 9. Comparison of relative deformations in height component between Borowa Gora and Jozefoslaw obtained from GNSS (daily solutions) and PSI data 


\section{Results of PSI vs results of GNSS}

Results of GNSS were compared with the results of PSI on a common time span, i.e. from September 2004 to April 2008. Deformations obtained from GNSS measurements were converted to the radar Line of Sight (LOS) direction. As both permanent GNSS stations are located on almost the same range of ascending Envisat scene the difference between incidence angles of both stations can be neglected. Relative deformations in LOS component determined on GNSS stations are within the range of accuracy of the GNSS technique (Cisak et al., 2014). Comparison of relative deformations in LOS component between Borowa Gora and Jozefoslaw obtained from GNSS (weekly solutions) and PSI (average) data are presented in Figure 10. Statistics of relative deformations in LOS component between Borowa Gora and Jozefoslaw obtained from GNSS and PSI data are given in Table 3.

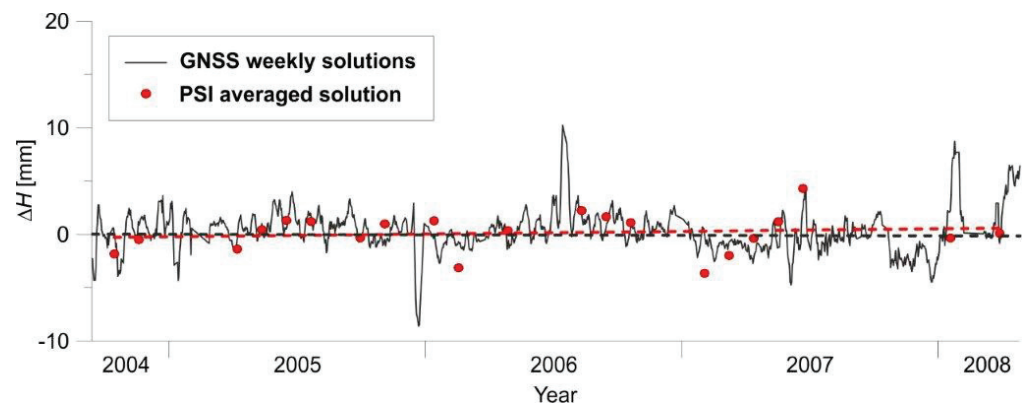

Fig. 10. Comparison of relative deformations in LOS component between Borowa Gora and Jozefoslaw obtained from GNSS (weekly solutions) and PSI (average) data

Table 3. Statistics of relative deformations in LOS component between Borowa Gora and Jozefoslaw obtained from GNSS and PSI data [mm]

\begin{tabular}{|l|c|c|c|}
\hline \multirow{2}{*}{} & \multirow{2}{*}{ PSI } & \multicolumn{2}{|c|}{ GNSS } \\
\cline { 3 - 4 } & & daily & weekly \\
\hline Mean & 0.1 & -0.1 & -0.1 \\
\hline Std. dev. & 1.8 & 2.9 & 1.9 \\
\hline Max-Min & 7.9 & 32.2 & 18.5 \\
\hline
\end{tabular}

The results of comparison of both methods do not show long periodic relative height variations in the period investigated. They show, however, short period (seasonal) variations which could be considered as the effect of hydrology. The results presented in Figure 10 and in Table 3 show good consistency of PSI results with GNSS results. They also indicate that PSI results can substantially increase reliability of the interpretation of the GNSS results (Hastaoglu, 2016). 
Differences between relative deformations in height component of BOGI and JOZ2 stations obtained from GNSS and PS and their statistics are given in Figure 11.

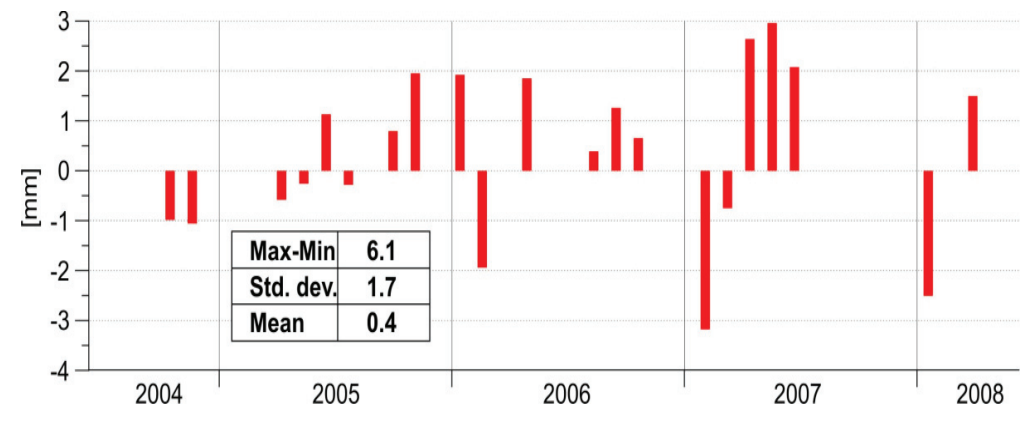

Fig. 11. Differences between relative deformations in height component of BOGI and JOZ2 stations obtained from GNSS and PS and their statistics

In the case of this study the best agreement of GNSS results with PSI results was obtained for epochs close to the reference epoch (2005-11-03). It is not surprising since the accuracy of PSI method decreases with growing distance from the reference point and from the reference epoch.

\section{Summary and conclusions}

The use of mutually complementing observation techniques for monitoring deformations in the investigated area, such as GNSS and PSI, can substantially increase reliability of the interpretation of the results obtained. Common use of both techniques allows in the variations considered as noise observation to extract the signal of height changes that is below the limit of measurement error and to ensure a proper absolute calibration of PSI data. Simultaneously, the analysis of results of two completely independent observation techniques leads to the increase of reliability of results obtained using each of these methods.

Archive radar data of a relatively small spatial resolution were used in this work. Also temporal resolution of the analysed data was rather small. The results obtained are, however, promising. It should be expected that comparing the results of the GNSS data with PSI data of higher spatial resolution, and in particular of higher temporal resolution, e.g. Cosmo-SkyMed or Sentinel-1 data, should give better results and allow to extract larger signal of height changes from the observed variations of vertical component of the GNSS station. 


\section{Acknowledgements}

Research was conducted in the framework of the project PBS2/B2/13/2014 "Integrated system of ground deformation monitoring with the use of satellite persistent scatterer interferometry (PSInSAR)" DefSAR financed by the National Centre of Science and Development, under the Programme for Applied Research coordinated by the Institute of Geodesy and Cartography, Warsaw, with the contribution of the Building Research Institute, Warsaw, and the Warsaw Surveying Company S.A.

\section{References}

Bosy, J. and Krynski, J. (2015). Reference frames and reference networks. Geodesy and Cartography, 64 (2), 5-29, DOI:10.1515/geocart-2015-0011

Bruyninx, C., Baire, Q., Legrand, J. and Roosbeek, F. (2011). The EUREF Permanent Network: Recent Developments and Key Issues. EUREF 2011 Symposium, Chisinau, Republic of Moldova, 25-28 May 2011. http://www.euref.eu/symposia/2011Chisinau/01-04-p-Bruyninx.pdf

Cisak, J., Godah, W. and Zak, L. (2011). Could recent GNSS data provide an evidence of tectonic processes within the TT zone?. EUREF 2011 Symposium, Chisinau, Republic of Moldova, 25-28 May $2011 \mathrm{http}: / /$ www.euref.eu/symposia/2011Chisinau/P-14-p-Cisak.pdf

Cisak, J., Zak, L., Stepniak, K., Wielgosz, P., Kupko, V.S., Olijnyk, A.E., Liubzhyn, A. and Zanimonskiy, Y.M. (2014). Investigation of uncertainty of GNSS-based distance metrology using EPN double stations data. EUREF 2014 Symposium, Vilnius, Lithuania, 4-6 June 2014. http://www.euref.eu/ symposia/2014Vilnius/P-10-Cisak.pdf

Dach, R., Hugentobler, U., Fridez, P. and Meindl, M. (Eds.) (2007). Bernese GPS Software Version 5.0. Astronomical Institute, University of Berne, Switzerland.

Feretti, A., Prati, C. and Rocca, F. (2000). Nonlinear subsidence rate estimation using permanent scatterers in differential SAR interferometry. IEEE Transactions on Geoscience and Remote Sensing, 38(5), 2202-2212.

Feretti, A., Prati, C. and Rocca, F. (2001). Permanent scatterers in SAR interferometry. IEEE Transactions on Geoscience and Remote Sensing, 39(1), 8-20.

Godah, W., Krynski, J. and Cisak, J., (2012). The use of GPS data at T-T Zone for the verification of the recent terrestrial reference frames considering possible geodynamic processes. Geoinformation Issues, 4, 1(4), 5-13.

Hastaoglu, K.O. (2016). Comparing the results of PSInSAR and GNSS on slow motion landslides, Koyulhisar, Turkey. Geomatics, Natural Hazards and Risk,7(2), 2016. DOI: http://dx.doi.org/10.1 080/19475705.2014.978822

Krynski, J. and Zanimonskiy, Y. (2005). Towards More Reliable Estimation of GPS Positioning Accuracy. A Window of the Future of Geodesy, IAG General Assembly, Sapporo, Japan, 30 June - 11 July 2003, Springer Verlag Berlin-Heidelberg, (ed.) F. Sansò, IAG Symposia, Vol. 128, 48-53.

Lyard, F., Lefevre, F., Letellier, T. and Francis, O. (2006). Modelling the global ocean tides: a modern insight from FES2004. Ocean Dynamics, 56, 394-415.

Massonet, D., Rossi, M., Carmona, C., Adagna, F., Peltzer, G., Feigl, K. and Rabaute, T. (1993). The displacement field of the Landers earthquake mapped by radar interferometry. Nature, 364(8), $138-142$.

Neill, A.E. (1996). Global mapping functions for the atmosphere delay at radio wavelengths. J. Geophys. Res., 101(B2), 3227-3246. 
Sawicki, L. (1960). The geological structure and morphology of the land in Warsaw (in Polish). Przeglad Geologiczny, 8 (12) 622.

Zebker, HA. and Goldstein, R.M. (1986). Topographic Mapping From Interferometric Synthetic Aperture Radar Observations. J. Geophys. Res., 91(B5), 4993-4999.

Zebker, H.A., Rosen, P.A., Goldstein, R.M., Gabriel, A. and Werner, C.L. (1994). On the derivation of coseismic displacement fields using differential radar interferometry: The Landers earthquake. J. Geophys. Res., 99(B10), 19617-19634.

http://www.epncb.oma.be/ 